\title{
Behavior-Based Acceleration Commanded Formation Flight Control
}

\author{
Touraj Soleymani ${ }^{1}$ and Fariborz Saghafi ${ }^{2}$ \\ Department of Aerospace Engineering, Sharif University of Technology, Tehran, Iran \\ ${ }^{1}$ soleymani@ae.sharif.edu \\ 2 saghafi@sharif.edu
}

\begin{abstract}
In this paper, the design of a formation flight controller is investigated. Each vehicle in the formation is controlled by designing two separate control loops. The formation flight controller placed in the outer loop employs behavior-based control as a distributed control strategy to steer the vehicle by producing acceleration commands and the control system placed in the inner loop is to convert these commands to the actuator commands. Leader following architecture is applied to define the structure for the formation flight. To study the pragmatic issues of the proposed formation flight controller, it is implemented into multiple micro air vehicles which are modeled by a six-degree-of-freedom dynamics. Finally, simulations were carried out to verify the effectiveness of the system.
\end{abstract}

Keywords: Formation Flight Control, Behavior-Based Control, Leader Following.

\section{INTRODUCTION}

The formation flight problem is an important research area concerning the coordination of multiple air vehicles. Flight of multiple air vehicles in formation with a defined geometry can provide many benefits, for instance, reduction of the fuel consumption due to decreasing the induced drag [1] and possibility of air refueling [2]. In order to maintain multiple autonomous air vehicles in formation, it is necessary to employ a formation flight controller. The notion of the formation control has been widely investigated in the literatures with the objectives of coordinating multiple robots [3], satellites [4], and aircraft [5]. Although the applications are different, the objectives are similar for the coordination of the multiple vehicles.

Behavior-based robotics is inspired from the intelligent behaviors of animals. A behavior is a mapping function producing responses from stimuli [6]. Behavior-based control is a decentralized control strategy used to autonomously control one robot or a group of robots. Note that a decentralized control system for an individual robot means that there is no planning or reasoning for the generation of responses. However, a decentralized or distributed control system for multi-robots indicates that there is no central part managing the system. In this study, the both notions are considered by employing the behavior-based control. In behavior-based control, the task is decomposed to the objective-oriented behaviors, which a robot requires to accomplish its objective [7]. Each behavior is independent and can have a direct access to the sensors and actuators of the robot. Behaviors operate in parallel to each other and their responses are coordinated to provide the agent's response. The emergent behavior which is an unpredictable behavior of the $\operatorname{robot}(\mathrm{s})$ is a consequence of local interactions between agents and the environment. Brooks [8] introduced the behavior-based control for controlling an autonomous mobile robot. Blach and Arkin [9] used the behavior-based control for formation of a multi robot team and Mataric [10] employed it to coordinate multi agents.

To construct the formation flight, it is required to determine the architecture of the formation. The leader following architecture is very popular for formation of multiple aircraft [11]. In this method, each vehicle is designated as a leader or/and follower. Then, each follower attempts to follow its leader by putting itself in a proper position with respect to the leader. In [12], two different structures are introduced for the leader following: leader mode and front mode. In the leader mode, there is solely one leader, and all followers are to follow the leader. In the front mode, each vehicle is to follow its front vehicle. Furthermore, in [13], two types of formation controller are presented. The first one is the $l-\psi$ controller and the second one is the $l-l$ controller. In the $l-\psi$ controller, each vehicle is to maintain a desired distance and a desired angle with respect to a leader, and in the $l-l$ controller, each vehicle is to keep two desired distances with respect to the two leaders which are specified for the vehicle.

In this study, the behavior-based method is exploited to generate the formation flight control commands since it is a distributed control strategy and the leader following method is applied to specify the architecture of the formation as it establishes a hierarchy in the group to form a structure.

Hereby, each vehicle in the formation is controlled by designing two separate control loops. The formation flight controller placed in the outer loop employs acceleration commands to steer the vehicles for maintaining the formation and the control system placed in the inner loop is to convert the guidance commands to the actuators commands. Designing the formation flight controller and the control system in the two separate loops and employing the acceleration commands in formation flight controller provide some advantages: employing a simple formation flight 
controller which can be implemented on any air vehicle with an arbitrary dynamics, providing the possibility of applying the traditional control theories in the inner loop, and dealing with load factor (acceleration) which better illustrates the constraints of a nonholonomic vehicle.

To model each vehicle in the formation, a six-degree-of-freedom dynamics of a micro air vehicle (MAV) is developed. Employing the six-degree-offreedom dynamics provide a high fidelity environment for investigation of the vehicle's constraints in the formation flight. Furthermore, MAVs which are small and highly maneuverable possess various advantages in urban areas.

The remainder of the paper is organized as follows. In section 2, the formation flight controller for a vehicle in the formation is presented by exploiting leader following architecture and behavior-based control. In section 3 , the consideration about control system of the vehicle is presented based on a bank-to-turn autopilot. Section 4 offers six-degree-of-freedom simulation results for the proposed formation flight controller, and finally section 5 remarks the conclusion.

\section{FORMATION FLIGHT CONTROLLER}

Formation flight controller is a high level controller which is placed in the outer control loop. Its objective is to generate guidance commands to steer the vehicles while providing a structured formation flight. The formation flight controller is constructed based on the behavior-based control by utilizing the leader following architecture. The formation flight controller commands are in terms of accelerations.

\subsection{Leader Following Architecture}

In this study, the leader following architecture is applied to specify the structure of the formation. The leader following is considered as a hierarchical structure for the formation flight problem. In this architecture, a vehicle is considered as a main leader carries the desired trajectory information and the other vehicles are selected as followers, each of which selects its front vehicle as the leader.

The formation flight structure can be represented by a graph. A graph $G$ is a pair $(V, E)$ that consists of a set of vertices $V=\{1,2, \ldots, n\}$ in correspondence with the $n$ vehicles in the formation and a set of edges $E \subseteq\{(i, j) \mid i, j \in V, i \neq j\}$ representing the existence of communication between each two vehicles. The graph $G$ is said to be directed if $(i, j) \in E<\neq>(j, i) \in E$. The input degree of the $k$ th vertex is defined the number of edges come in to it and the output degree of the $k$ th vertex is defined the number of edges go out of it.

Accordingly, each vehicle has a specific input and output degrees. The vehicle, whose input degree is zero, is located at the top of the hierarchy and considered as the main leader and specifies the desired trajectory. The vehicles whose input and output degrees are greater that one perform both functions of a leader and a follower and are placed at the middle of the hierarchy, and the vehicles whose output degree is zero solely perform the function of a follower and are located at the bottom of hierarchy. The leader following architecture for V-shape formation is illustrated in Fig. 1.

Note that the formation flight controller is implemented in each follower vehicle so in the subsequent sections the derivation is confined for a representative vehicle. It is also assumed that a follower can acquire the information about the inertial displacement and velocity of itself via IMU and its leader via communication.

Furthermore, the neighbor set of the vertex $i$ is defined as $N_{i}=\left\{(i, j)|| s_{j i} \mid<d_{N}\right\}$ which is specified by a radius distance $d_{N}$. It is supposed that neighbors share their inertial displacement and velocity information with each other via communication.

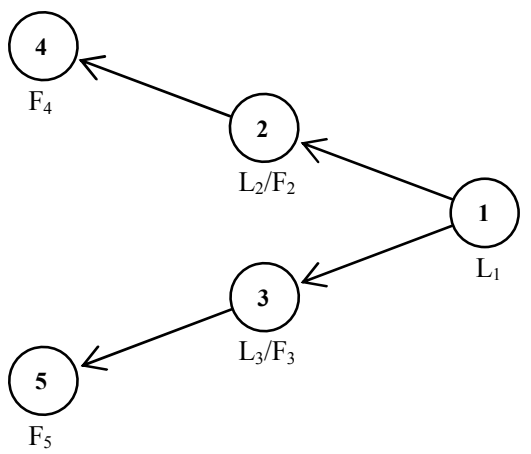

Figure 1. Leader following architecture.

\subsection{Behavior-Based Control}

The behavior-based method is exploited to generate the formation flight controller commands. In this approach, corresponding to the objective, the behaviors are required to be defined. Each behavior is defined by a mathematical function called rule. The resultant response in each vehicle is made by combining the responses of these behaviors. The formation flight is a result of local interactions between the vehicles and environment. The behaviors used in this study are distance adjustment, velocity alignment, structure forming, and collision avoidance.

Fig. 2 thoroughly illustrates a portion of the formation flight which consists of a follower together with its leader, where $F$ is the base point and $\mathbf{f}_{\mathbf{1}}, \mathbf{f}_{\mathbf{2}}$, and $\mathbf{f}_{3}$ are the three base vectors of the follower frame, $L$ is the base point and $\mathbf{l}_{\mathbf{1}}, \mathbf{l}_{\mathbf{2}}$, and $\mathbf{l}_{\mathbf{3}}$ are the three base vectors of the leader frame, $E$ is the base point and $\mathbf{e}_{1}, \mathbf{e}_{2}$, and $\mathbf{e}_{3}$ are the three base vectors of the inertial frame, $\mathbf{s}_{\mathbf{F E}}$ and $\mathbf{s}_{\mathbf{L E}}$ are the displacement vectors of the follower and the leader with respect to the inertial reference point respectively, $\mathbf{s}_{\mathbf{L F}}$ is the displacement vector of the leader with respect to the follower, $\mathbf{v}_{\mathbf{F}}^{\mathbf{E}}$ and $\mathbf{v}_{\mathbf{L}}^{\mathbf{E}}$ are the velocity vectors of the follower and the leader with respect to the inertial reference frame respectively, and $\mathbf{V}_{\mathbf{L F}}^{\mathbf{E}}$ is inertial differential velocity of the leader with respect to the 
follower. Notice, throughout this paper, the body coordinate system of the follower and the local level coordinate system are represented as $[.]^{F}$ and $[.]^{L}$ respectively. To define a coordinate system, its axes are required to be specified. For a general right-handed Cartesian coordinate system $[.]^{X}$, the axes are represented as $1^{X}, 2^{X}$, and $3^{X}$. Note that $B_{i}$ and $[.]^{B_{i}}$ generally represents $i$ th vehicle in the formation and its coordinate system respectively, regardless of the leader following architecture. The symbols used in this paper are mainly quoted from [14].

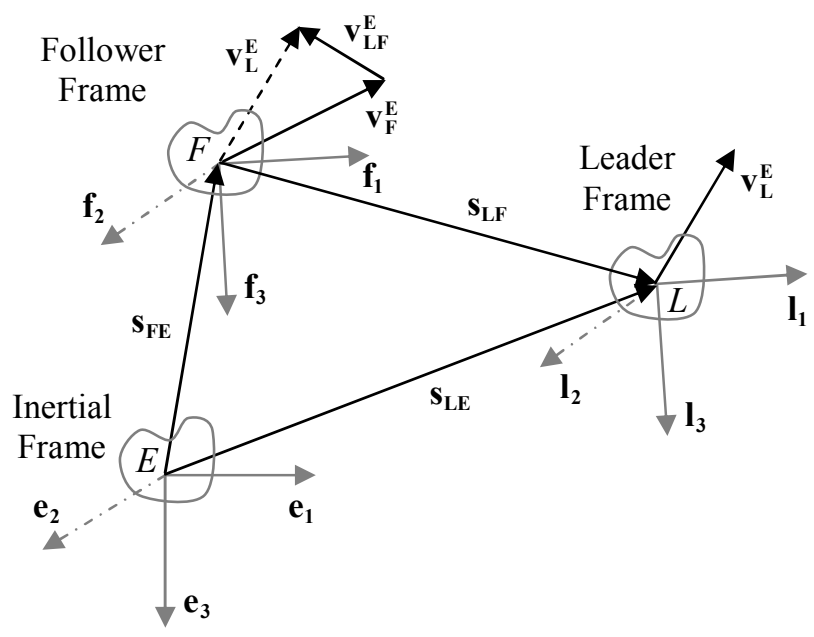

Figure 2. Geometry of a follower and its leader.

\subsubsection{Distance Adjustment Behavior}

In order to adjust an equilibrium distance with respect to the leader, the distance adjustment behavior is used by the follower. The rule of this behavior generates an acceleration command which is a function of the displacement vector of the leader with respect to the follower. This command expressed in the local level coordinate system is written as

$$
\left[a_{1}\right]^{L}=\frac{f_{1}\left(\left|s_{L F}\right|-d_{e}\right)}{\left|s_{L F}\right|}\left[s_{L F}\right]^{L}
$$

where $|$.$| represents the magnitude of a vector, \mathbf{a}_{\mathbf{1}}$ is the acceleration command belonging to the distance adjustment behavior, $f_{1}$ is a control function, $d_{e}$ is the equilibrium distance, and $\mathbf{s}_{\mathbf{L F}}$ can be calculated assuming the follower can obtain its inertial displacement vector and that of its leader as

$$
\left[s_{L F}\right]^{L}=\left[s_{L E}\right]^{L}-\left[s_{F E}\right]^{L}
$$

The acceleration command $\mathbf{a}_{\mathbf{1}}$ is in the direction of $\mathbf{s}_{\mathbf{L F}}$ and has the magnitude of $f_{1}\left(\left|s_{L F}\right|-d_{e}\right)$. Note that $f_{1}$ specifies the quickness of the formation flight system response.

\subsubsection{Velocity Alignment Behavior}

To align the velocity of the follower with the velocity of the leader, the velocity alignment behavior is used. The rule of this behavior generates an acceleration command which is a function of the inertial differential velocity of the leader with respect to the follower. This command expressed in the local level coordinate system is written as

$$
\left[a_{2}\right]^{L}=\frac{f_{2}\left(\left|v_{L F}^{E}\right|\right)}{\left|v_{L F}^{E}\right|}\left[v_{L F}^{E}\right]^{L}
$$

where $\mathbf{a}_{2}$ is the acceleration command belonging to the velocity alignment behavior, $f_{2}$ is a control function, and $\mathbf{v}_{\mathbf{L F}}^{\mathbf{E}}$ can be calculated supposing the follower can obtain its inertial velocity and that of the leader as

$$
\left[v_{L F}^{E}\right]^{L}=\left[v_{L}^{E}\right]^{L}-\left[v_{F}^{E}\right]^{L}
$$

The acceleration command $\mathbf{a}_{2}$ is in the direction of $\mathbf{v}_{\mathbf{L F}}^{\mathbf{E}}$ and has the magnitude of $f_{2}\left(\left|v_{L F}^{E}\right|\right)$. Note that $f_{2}$ determines the damping characteristics of the system. In fact, in the perspective of the control theory, this leads to an axial damping works in the direction of the line of sight (LOS), i.e. the line that connects the follower to the leader, and a side damping operates perpendicular to the LOS. This causes the velocity of the leader to be followed by the follower.

\subsubsection{Structure Forming Behavior}

In spite of adjusting the relative distances and aligning the velocities of the followers with respect to the leaders, it is desired to construct a structure for the formation flight. In order to construct a structure, another behavior must be used to fix the position of the followers with respect to the leaders. For this purpose, the follower employs two angles, azimuth and elevation. The azimuth and elevation angles are defined as the angles which $\mathbf{s}_{\mathbf{L F}}$ makes with respect to a reference coordinate system. The velocity coordinate system of the leader is considered as the reference coordinate system. Note the velocity coordinate system of the leader is a coordinate system in which the $1^{V_{L}}$ axis is parallel and in the direction of $\mathbf{v}_{\mathbf{L}}^{\mathbf{E}}$, the $2^{V_{L}}$ axis is perpendicular to the $1^{V_{L}}$ axis and remains in the local horizontal plane, and the $3^{V_{L}}$ axis is perpendicular to $1^{V_{L}}$ and $2^{V_{L}}$ such that completes the right-handed coordinate system. The tangential plane is defined as a plane which consists of $1^{V_{L}}$ and $2^{V_{L}}$.

Consequently, in order to calculate these two angles, it is necessary to obtain $\left[s_{L F}\right]^{V_{L}}$, where $[.]^{V_{L}}$ represents the velocity coordinate system of the leader. Since $\left[v_{L}^{E}\right]^{L}$ are acquired by the follower, it can be written in terms of its components as

$$
\left[\bar{v}_{L}^{E}\right]^{L}=\left[\begin{array}{lll}
u_{L} & v_{L} & w_{L}
\end{array}\right]
$$

Using the components of $\left[v_{L}^{E}\right]^{L}$, the heading and flight-path angles of the leader can be calculated as

$$
\begin{gathered}
\chi=\operatorname{atan}\left(\frac{v_{L}}{u_{L}}\right) \\
\gamma=\operatorname{atan} 2\left(\frac{-w_{L}}{\sqrt{u_{L}^{2}+v_{L}^{2}}}\right)
\end{gathered}
$$

Note that the outputs of atan and atan2 are $[-\pi / 2, \pi / 2]$ and $[-\pi, \pi]$ respectively. In this case the whole points in space are covered. 
The transformation matrix between the leader velocity coordinate system and the local level coordinate system can be written in terms of the heading and flight-path angles of the leader as

$$
[T]^{V_{L} L}=\left[\begin{array}{ccc}
\cos \gamma \cos \chi & \cos \gamma \sin \chi & -\sin \gamma \\
-\sin \chi & \cos \chi & 0 \\
\sin \gamma \cos \chi & \sin \gamma \sin \chi & \cos \gamma
\end{array}\right]
$$

Therefore, $\left[s_{L F}\right]^{V_{L}}$ can be calculated as

$$
\left[s_{L F}\right]^{V_{L}}=[T]^{V_{L} L}\left[s_{L F}\right]^{L}
$$

One can represent $\left[s_{L F}\right]^{V_{L}}$ in terms of its components such as

$$
\left[\bar{s}_{L F}\right]^{V_{L}}=\left[\begin{array}{lll}
x_{L F} & y_{L F} & z_{L F}
\end{array}\right]
$$

The azimuth and elevation angles which specify the position of the follower with respect to the leader, can be computed by using the components of $\left[s_{L F}\right]^{V_{L}}$ as

$$
\begin{gathered}
A z=\operatorname{atan}\left(\frac{y_{L F}}{x_{L F}}\right) \\
E l=\operatorname{atan} 2\left(\frac{-z_{L F}}{\sqrt{x_{L F}^{2}+y_{L F}^{2}}}\right)
\end{gathered}
$$

where $A z$ and $E l$ represent the azimuth and elevation angles respectively.

The rule of the structure forming behavior generates an acceleration command attempting to track the desired azimuth and elevation angles in order to construct a structure for the formation flight. This command expressed in the local level coordinate system is written as

$$
\left[a_{3}\right]^{L}=f_{3}\left(A z-A z_{c}\right)\left[e_{A z}\right]^{L}+f_{4}\left(E l-E l_{c}\right)\left[e_{E l}\right]^{L}
$$

where $\mathbf{a}_{3}$ is the acceleration command belonging to the structure forming behavior, $f_{3}$ and $f_{4}$ are control functions, $A z_{c}$ and $E l_{c}$ are the command azimuth and elevation angles, $\mathbf{e}_{\mathrm{Az}}$ is the unit vector perpendicular to projection of $\mathbf{s}_{\mathbf{L F}}$ into the tangential plane and $\mathbf{e}_{\mathbf{E I}}$ is the unit vector perpendicular to the tangential plane. The projection of $\mathbf{s}_{\mathbf{L F}}$ into the tangential plane is calculated as

$$
\left[s_{L F_{P}}\right]^{V_{L}}=\left([E]^{V_{L}}-[n]^{V_{L}}[\bar{n}]^{V_{L}}\right)\left[s_{L F}\right]^{V_{L}}
$$

where $[E]^{V_{L}}$ is the identity matrix and $[\bar{n}]^{V_{L}}=\left[\begin{array}{lll}0 & 0 & 1\end{array}\right]$ is the unit vector normal to the tangential plane.

The unit vectors, $\mathbf{e}_{\mathrm{Az}}$ and $\mathbf{e}_{\mathrm{El}}$, can be written in the velocity coordinate system of the leader as

$$
\begin{gathered}
{\left[e_{A z}\right]^{V_{L}}=\frac{\left[S_{L F_{P}}\right]^{V_{L}}[n]^{V_{L}}}{\left|s_{L F_{P}}\right|}} \\
{\left[e_{E l}\right]^{V_{L}}=[n]^{V_{L}}}
\end{gathered}
$$

where $\left[S_{L F_{P}}\right]^{V_{L}}$ is the skew symmetric matrix of $\left[s_{L F_{P}}\right]^{V_{L}}$.

Since $\mathbf{e}_{\mathrm{Az}}$ and $\mathbf{e}_{\mathrm{El}}$ should be expressed in the local level coordinate system, they must be transformed by using the $[T]^{V_{L} L}$ as follow

$$
\begin{aligned}
{\left[e_{A z}\right]^{L} } & =[\bar{T}]^{V_{L} L}\left[e_{A z}\right]^{V_{L}} \\
{\left[e_{E l}\right]^{L} } & =[\bar{T}]^{V_{L} L}\left[e_{E l}\right]^{V_{L}}
\end{aligned}
$$

\subsubsection{Collision Avoidance Behavior}

In order to avoid collisions in the formation between any two vehicles, the collision avoidance behavior is used. This behavior generates an acceleration command in terms of displacement vectors of the neighbors with respect to the follower. This command expressed in the local level coordinate system is written as

$$
\left[a_{4}\right]^{L}=\sum_{j \in N_{F}} \frac{f_{5}\left(\left|s_{B_{j} F}\right|-d_{N}\right)}{\left|s_{B_{j} F}\right|}\left[s_{B_{j} F}\right]^{L}
$$

where $N_{F}$ is the neighbor set of the follower, $\mathbf{a}_{4}$ is the acceleration command belonging to the collision avoidance behavior, $f_{5}$ is a control function, and $\mathbf{s}_{\mathbf{B}_{\mathbf{B}} \mathbf{F}}^{\mathbf{E}}$ is the relative displacement vector of the neighbors with respect to the follower. Assuming that the follower can obtain the displacement of its neighbors, $\mathbf{S}_{\mathbf{B}_{\mathbf{j}} \mathbf{F}}^{\mathbf{E}}$ is calculated as

$$
\left[s_{B_{j} F}\right]^{L}=\left[s_{B_{j} E}\right]^{L}-\left[s_{F E}\right]^{L}
$$

Note that this behavior only produces repulsive commands to move the vehicle away from the colliding vehicles since the equilibrium distance used in the control function $f_{5}$ is the same as the neighborhood radius defined before, $d_{N}$.

\subsubsection{Resultant Response}

In order to construct the resultant response for each follower, the responses of the behaviors must be coordinated. Therefore, the four generated acceleration command are combined as

$$
[a]^{L}=\left[a_{1}\right]^{L}+\left[a_{2}\right]^{L}+\left[a_{3}\right]^{L}+\left[a_{4}\right]^{L}
$$

In this study, the control functions used in the behavior rules are considered as simple constant gains. In order to prevent big acceleration commands, command saturation is taken into account for each behavior. The saturation function can be written as

$$
\text { sat }\left(\left|a_{i}\right|\right)=\left\{\begin{array}{cc}
0 & x>\left|a_{u_{i}}\right| \\
\left|a_{i}\right| & \left|a_{l_{i}}\right|<x<\left|a_{u_{i}}\right| \\
0 & x>\left|a_{l_{i}}\right|
\end{array}\right.
$$

where index $i$ indicates the behavior, $\left|a_{u_{i}}\right|$ and $\left|a_{l_{i}}\right|$ are the upper and lower bands for $i$ th acceleration command respectively. Therefore, the $i$ th acceleration command is expressed as

$$
\left[a_{i}\right]^{L}=\operatorname{sat}\left(\left|a_{i}\right|\right) \frac{\left[a_{i}\right]^{L}}{\left|a_{i}\right|}
$$

\section{VEHICLE CONTROL SYSTEM}

In order to execute the generated acceleration commands, a control system is used to convert them into the MAV's control surface commands. Thus, the vehicle control system is a low level controller which is placed in the inner loop. Although the focus of this paper is not on the vehicle control system but there are some consideration about its design

To consider the ballistic part of the acceleration, the acceleration command produced by formation flight 
controller, a , must be compensated by the gravity acceleration. This can be written as

$$
\left[a_{\text {comp }}\right]^{L}=[a]^{L}-[g]^{L}
$$

where $\mathbf{a}_{\text {comp }}$ is the compensated acceleration command and $\mathbf{g}$ is the gravity acceleration vector.

Inasmuch as the acceleration command is expressed in the local level coordinate system, it must be transformed to the body coordinate system. This is done by using the transformation matrix between the body coordinate system and the local level coordinate system

$$
\left[a_{\text {comp }}\right]^{B_{i}}=[T]^{B_{i} L}\left[a_{\text {comp }}\right]^{L}
$$

where $[T]^{B_{i} L}$ is the transformation matrix between the body coordinate system and the local level coordinate system.

Since the produced acceleration command is suitable for skid-to-turn (STT) vehicles it is required to be converted to an acceleration which can be used by the MAV as a bank-to-turn (BTT) vehicle. The STT acceleration command can be expressed as

$$
\left[\bar{a}_{\text {comp }}\right]^{B_{i}}=\left[\begin{array}{lll}
a_{x}^{S} & a_{y}^{S} & a_{z}^{S}
\end{array}\right]
$$

By applying the conversion between the STT and the BTT coordinates, roll command and acceleration command for a BTT vehicle is derived as

$$
\begin{gathered}
\Delta \phi=\tan ^{-1}\left(\frac{-a_{y}^{S}}{a_{z}^{S}}\right) \\
a^{B}=\operatorname{sgn}\left(a_{z}^{S}\right) \sqrt{\left(a_{y}^{S}\right)^{2}+\left(a_{z}^{S}\right)^{2}}
\end{gathered}
$$

where $\Delta \phi$ is the BTT error roll command, $a^{B}$ is the BTT acceleration command, and sgn is the sign function.

The control system was designed in four channels of roll, pitch, yaw, and axial.

\section{SIMULATION}

To verify the performance of the system, a six-degree-of-freedom simulation for a scenario was developed by using MATLAB ${ }^{\circledR} /$ Simulink. The scenario constitutes of five MAVs which are to fly in formation. The general specifications of each vehicle are as follow: $m=0.445 \mathrm{~kg}, I_{x x}=0.002 \mathrm{~kg} . \mathrm{m}^{2}, I_{y y}=0.008 \mathrm{~kg} . \mathrm{m}^{2}$, $I_{z z}=0.01 \mathrm{~kg} \cdot \mathrm{m}^{2}, \quad I_{x z}=2.10 e-4 \mathrm{~kg} . \mathrm{m}^{2}, \quad b=0.61 \mathrm{~m}$, $S=0.089 \mathrm{~m}^{2}, \quad \bar{c}=0.147 \mathrm{~m} \quad, \quad V_{\text {cuise }}=15 \mathrm{~m} / \mathrm{s}$, $T_{\max }=3.2 \mathrm{~N}$, the control surface deflections are $\pm 15 \mathrm{deg}$, and the control surface rate limits are considered $\pm 50 \mathrm{deg} / \mathrm{s}$. The initial conditions of these vehicles are introduced as

$$
\begin{aligned}
& {\left[\bar{s}_{B_{1} E}\right]^{L}=\left[\begin{array}{lll}
0 & 100 & -100
\end{array}\right] ;\left[\bar{v}_{B_{1} E}^{B_{1}}\right]^{B_{1}}=\left[\begin{array}{lll}
15 & 0 & 0
\end{array}\right]} \\
& {\left[\bar{s}_{B_{2} E}\right]^{L}=\left[\begin{array}{lll}
-20 & 95 & -90
\end{array}\right] ;\left[\bar{v}_{B_{2}}^{E}\right]^{B_{2}}=\left[\begin{array}{lll}
16 & 0 & 0
\end{array}\right]} \\
& {\left[\bar{s}_{B_{3} E}\right]^{L}=\left[\begin{array}{lll}
-20 & 105 & -85
\end{array}\right] ;\left[\bar{v}_{B_{3}}^{E}\right]^{B_{3}}=\left[\begin{array}{lll}
16 & 0 & 0
\end{array}\right]} \\
& {\left[{\overline{s_{B} E}}_{B_{4}}\right]^{L}=\left[\begin{array}{lll}
-35 & 70 & -85
\end{array}\right] ; \quad\left[\bar{v}_{B_{4}}^{E}\right]^{B_{4}}=\left[\begin{array}{lll}
15.5 & 0 & 0
\end{array}\right]} \\
& {\left[\bar{s}_{B_{5} E}\right]^{L}=\left[\begin{array}{lll}
-40 & 130 & -90
\end{array}\right] ;\left[\bar{v}_{B_{5}}^{E}\right]^{B_{5}}=\left[\begin{array}{lll}
15.5 & 0 & 0
\end{array}\right]}
\end{aligned}
$$

The desired trajectory of the formation flight is specified by the main leader, $B_{1}$. This is a straight and level flight which is defined as

$$
\left[\bar{s}_{B_{1} E}(t)\right]^{L}=\left[\begin{array}{lll}
15 t & 0 & 0
\end{array}\right]
$$

Here, the desired shape of the formation flight is $\mathrm{V}$-shape formation. The equilibrium distance between a follower and its leader is considered $10 \mathrm{~m}$, the desired azimuth and elevation angles for $B_{2}$ and $B_{4}$ are considered $20 \mathrm{deg}$ and $0 \mathrm{deg}$ respectively, and the desired azimuth and elevation angles for $B_{3}$ and $B_{5}$ are considered $-20 \mathrm{deg}$ and $0 \mathrm{deg}$ respectively.

The 3D trajectories of the five vehicles in the formation flight are shown in Fig. 3. The magnitude of the relative distance between each follower and its leader is illustrated in Fig. 4. It can be seen that the relative distances of all followers converge to the equilibrium distance. Fig. 5 illustrates the magnitude of the inertial differential velocity of each follower with respect to its leader. It is shown that the magnitudes of inertial differential velocities of all followers converge to zero. The azimuth and elevation angles of each follower with respect to its leader are illustrated in Fig. 6 and Fig. 7 respectively. It can be seen that these angles converge to their desired angles for each follower.

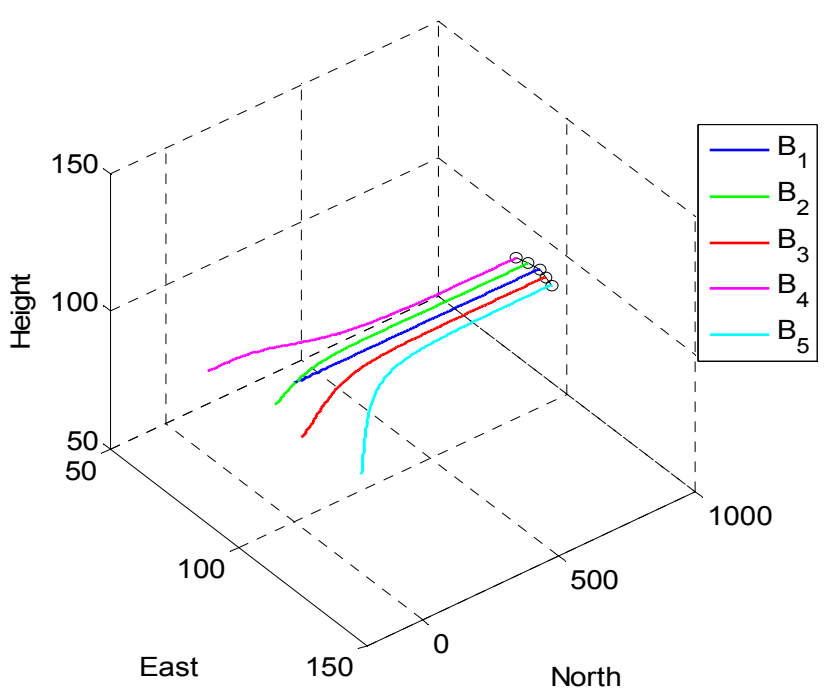

Figure 3. Trajectories of the five vehicles.

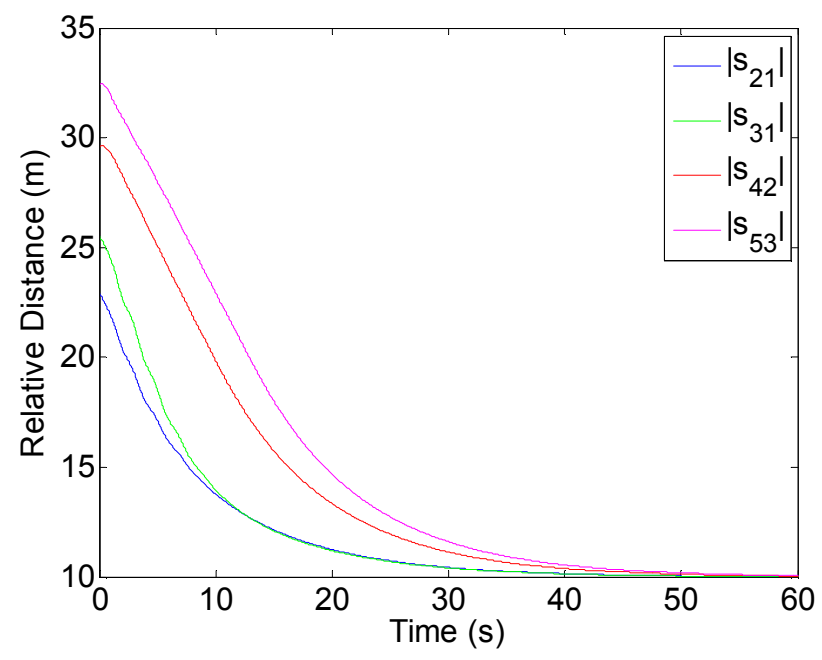

Figure 4. Magnitudes of relative displacements of followers. 


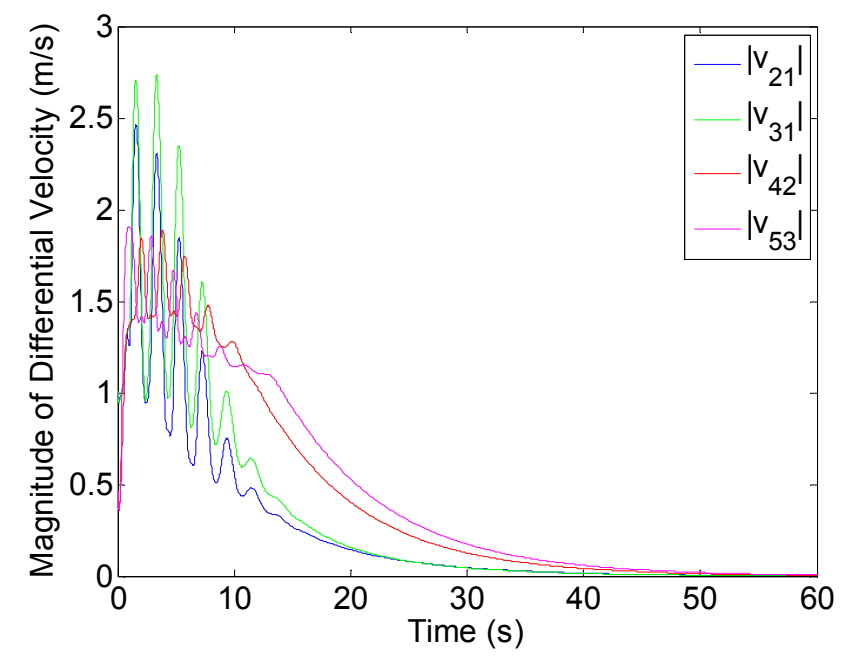

Figure 5. Magnitudes of differential velocities of followers.

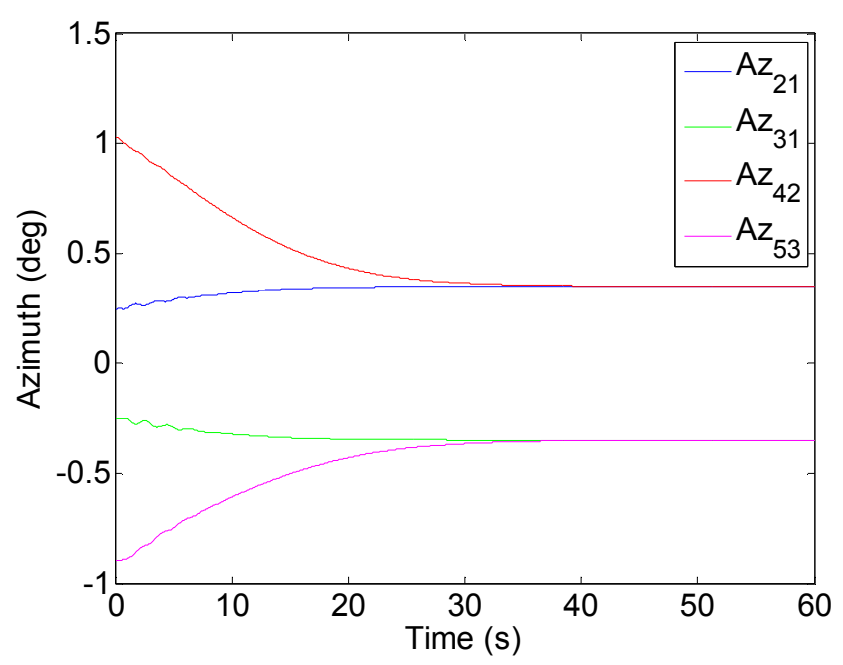

Figure 6. Azimuth angles of followers.

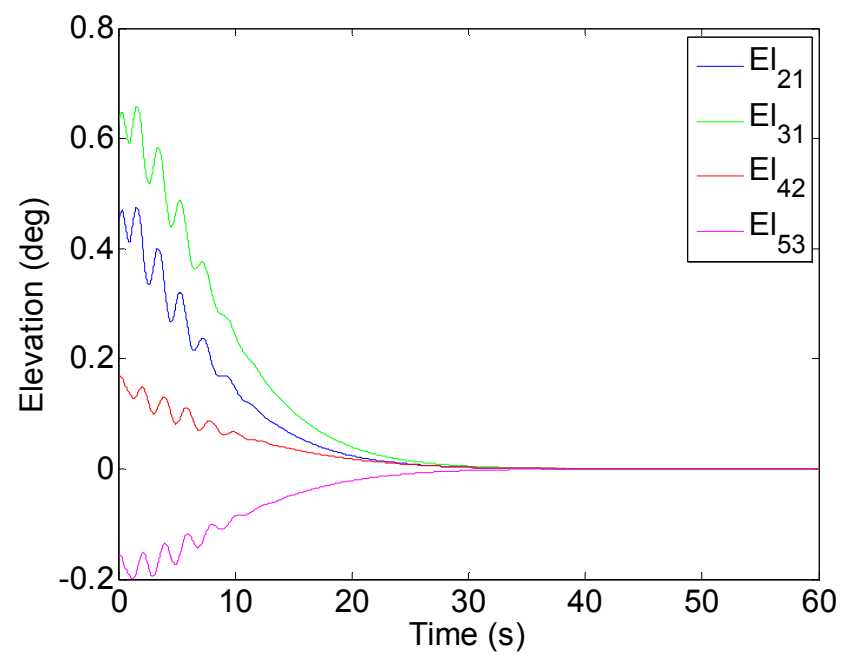

Figure 7. Elevation angles of followers.

\section{CONCLUSION}

In this study, the formation flight control was investigated. Leader following architecture specified the structure of the formation. Formation flight controller was designed by employing behavior-based control strategy. Four behaviors were introduced and then the resultant response was produced by combining the responses of these behaviors. The formation flight controller was implemented on the MAVs which were equipped by bank-to-turn autopilots. The six-degreeof-freedom simulations were carried out to show the performance of the system. The results demonstrated that followers are able to converge to the formation flight; specifically, each follower adjusts its relative distance with respect to its leader, aligns its velocity with the velocity of its leader, forms a specific structure of the formation by adjusting the two reference angles with respect to its leader, and avoids the collisions.

\section{REFERENCES}

[1] E. Lavretsky, "F/A-18 Autonomous Formation Flight Control Systems Design," AIAA 2002-4757.

[2] Nalepka, Joesph P. and Jacob L. Hinchman. Automated AerialRefueling: Extending the Effectiveness of Unmanned Air Vehicles, Air Force Research Laboratory (AFRL), AIAA 2005.

[3] P.K.C. Wang, "Neural Network Output Feedback Control of Robot Formations," IEEE Transactions on System, Man, and Cybernetics, Vol. 40, No. 2, 2010.

[4] J. Shan, "6-DOF Synchronization Control for Multiple Spacecraft Formation Flying," AIAA 2008-6468.

[5] Y. Gu, B. Seanor, G. Campa, M. R. Napolitano, L. Rowe, S Gururajan, and S. Wan, "Design and Flight Testing Evaluation of Formation Control Laws," IEEE Transactions on Control System Technology, Vol. 14, No. 6, 2006.

[6] R. C. Arkin, "Behavior-Based Robotics," MIT press, 1998.

[7] D. Floreano and C. Mattiussi, "Bio-Inspired Artifical Intelligence," MIT press, 2008.

[8] R. Brook, "A Robust Layered Control System for a Mobile Robot," IEEE Journal of Robotics and Automation, Vol. RA-2, No. 1, 1986.

[9] T. Blach and R.C. Arkin, "Behavior-Based Formation Control for multirobot teams," IEEE Transactions on Robotics and Automation, Vol. 14, No. 6, 1998.

[10] M. J. Mataric, "Behavior-Based Control: Examples from Navigation, Learning, and Group Behavior," Journal of Experimental and Theoretical Artificial Intelligence, Vol. 9, 1997.

[11] A.K. Das, R. Fierro, V. Kumar, J.P. Ostrowski, J. Spletzer, and C.J. Taylor, "A Vision-Based Formation Control Framework," IEEE Transaction on Robotics and Automation, Vol. 18, No. 5, 2002

[12] F. Giulietti, L. Pollini, and M. Innocent, "Autonomous Formation Flight," IEEE Control Systems Magazine, Vol. 20, No. 6, 2000.

[13] J. P. Desai, J. Ostrowski, and V. Kumar, "Controlling Formations of Multiple Mobile robots," Proceedings of IEEE International Conference on Robotics and Automation, May 1998.

[14] P. Zipfel, "Modeling and Simulation of Aerospace Vehicles Dynamics," AIAA Education Series, Second Edition, 2007. 